\title{
Review of: "Genetic profiles of Barrett's esophagus and esophageal adenocarcinoma in Japanese patients"
}

Isamu Hoshino

Potential competing interests: The author(s) declared that no potential competing interests exist.

IIn this study, the development of Barrett's esophageal cancer (EAC) was examined by dividing the Barrett's esophagus into a Short Segment Barrett's esophagus of $3 \mathrm{~cm}$ or less (SSBE), a Long Segment Barrett's esophagus of $3 \mathrm{~cm}$ or more (LSBE), and early Barrett's esophageal cancer. The gene expression profile is being examined using the Next generation sequencer. The driver mutation including P53 is not high in $\mathrm{BE}$, but once it develops, the frequency of mutation increases in BE around the cancer. It is a very interesting research content. However, as they also mention in the limitation, special attention should be paid to the place where the sample was collected by LSBE. It is presumed that the site where EAC occurs is relatively rare at the tip of $\mathrm{BE}$. In fact, verification by multisampling at multiple locations, especially in LSBE, is desired. In addition, it is undeniable that the number of cases is small and the study is primitive. We believe that verification with an increased number of cases in collaboration with other institutions will make their results more robust. 